\title{
Upper extremity fractures due to intimate partner violence versus accidental causes
}

\author{
Bharti Khurana ${ }^{1}\left(\right.$ D Ali Raja $^{2} \cdot$ George S. M. Dyer ${ }^{3} \cdot$ Steven E. Seltzer ${ }^{4} \cdot$ Giles W. Boland $^{4} \cdot$ Mitchel B. Harris $^{5}$. \\ Paul Tornetta $^{6} \cdot$ Randall T. Loder $^{7}$
}

Received: 21 June 2021 / Accepted: 15 July 2021 / Published online: 9 October 2021

(c) American Society of Emergency Radiology 2021

\begin{abstract}
Purpose The purpose of this study is to evaluate the prevalence of intimate partner violence (IPV)-related upper extremity fractures (UEF) in women presenting to US emergency departments (ED) and compare their anatomic location to those due to accidental falls or strikes.

Methods An Institutional Review Board exempt, retrospective review of prospectively collected data was performed using the National Electronic Injury Surveillance System's All Injury Program data from 2005 through 2015 for all UEF sustained in women 15 to 54 years old. Injuries based on reported IPV versus accidental falls or strikes were analyzed accounting for the weighted, stratified nature of the data.

Results IPV-related UEF represented $1.7 \%$ of all UEF and $27.2 \%$ of all IPV fractures. The finger was the most common fracture site in IPV (34.3\%) and accidental striking (53.3\%) but accounted for only $10 \%$ of fall-related UEF. There was a higher proportion of shoulder fractures in IPV (9.2\%) compared to accidental falls (7.4\%) or strikes (2.9\%). The odds of a finger fracture were 4.32 times greater in IPV than falling and of a shoulder fracture were 3.65 greater in IPV than accidental striking $(p<0.0001)$.

Conclusions While the finger is the most common site for IPV UEF, it is also the most common location for accidental striking. A lower proportion of finger fractures in fall and of shoulder/forearm fractures in accidental striking should prompt the radiologist to discuss the possibility of IPV with the ED physician in any woman presenting with a finger fracture due to fall and a shoulder/forearm fracture with a vague history of accidental striking.
\end{abstract}

Keywords Intimate partner violence $\cdot$ Upper extremity fractures $\cdot$ Finger fractures

The work in this manuscript has never been presented or published elsewhere.

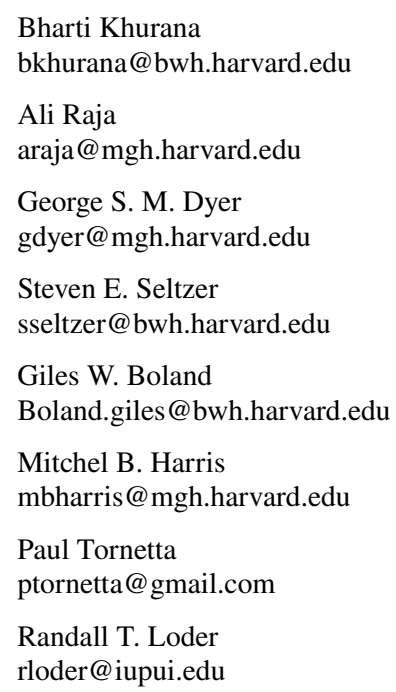

1 Trauma Imaging Research and Innovation Center, Division of Emergency Radiology, Brigham and Women's Hospital, Boston, MA 02115, USA

2 Department of Emergency Medicine, Massachusetts General Hospital, Boston, MA 02115, USA

3 Department of Orthopedic Surgery, Brigham and Women's Hospital, Harvard Medical School, 75 Francis Street, Boston, MA 02115, USA

4 Brigham and Women's Hospital, Boston, MA 02115, USA

5 Department of Orthopedic Surgery, Massachusetts General Hospital, Boston, MA 02114, USA

6 Department of Orthopedics, Boston Medical Center, Boston, MA, USA

7 Riley Children's Hospital, ROC 4250, 705 Riley Hospital Drive, Indianapolis, IN 46202, USA 


\section{Background}

Intimate partner violence (IPV), defined as physical, sexual, or emotional violence between current or former partners, is a significant public health issue affecting nearly one out of three US women [1]. According to National Intimate Partner and Sexual Violence Survey (NISVS), one in five women has reported experiencing severe physical violence from an intimate partner in their lifetime. However, IPV remains profoundly underdiagnosed because only $2.5-15 \%$ of IPV victims self-disclose [2]. The emergency department (ED) is frequently the first point of care for victims of IPV experiencing acute injuries [3]. Each year, one in two female homicides is linked to IPV [4], and nearly half of female homicide victims present to the ED within 2 years before the homicide [5]. While injuryrelated ED visits provide trauma and emergency healthcare providers a unique opportunity to diagnose IPV [6], it has been reported that nearly half of the IPV victims present to ED with symptoms not directly related to violence, emphasizing rigorous screening [7]. Despite screening questions often used during ED triage, IPV often goes undetected [8]. Unlike nonaccidental trauma in children, the nature and pattern of IPV injuries are not well described [7, 9]. As a result, acute care healthcare providers, especially those specializing in trauma, emergency medicine, orthopedic surgery, plastic surgery, and radiology, focus their care on treating the injuries and not on the underlying etiology of the injury $[10,11]$. Although the incidence of physical IPV has increased during the COVID-19 pandemic [12], there is little published data on ways in which IPV victims can be identified proactively [13]. Knowledge of injury patterns specific to IPV may help emergency clinicians and subsequent follow-up providers raise concern for IPV and provide timely resources to victims, thereby preventing additional cycles of abuse, life-threatening injuries, and potential homicides [14].

By establishing characteristic injury patterns linked to IPV, an objective assessment of IPV can be made instead of solely relying on victim self-report [15]. Upper extremity fractures (UEF) are the second most common IPVrelated fracture location after craniofacial fractures [16, 17]. One in six women presenting to an orthopedic clinic with an extremity injury have a history of abuse in the past year [18], yet to the authors' knowledge, there have been only a few studies to differentiate IPV-related extremity fractures from those sustained due to other causes [19, 20]. UEF are often sustained in defense when the victim tries to protect the central body or face from the abuser [20]. Previous studies on smaller subsets of patients have described the finger as the most common IPV UE fracture site $[17,19,21]$. However, it is not clear if the location of
UEF in IPV victims is different from the general accidental trauma population, where the most common mechanisms are a fall or accidental striking (excluding motor vehiclerelated causes).

We hypothesized that the different mechanism of injury leads to different fracture locations, allowing the clinician to recognize IPV, compared to non-IPV etiologies, based on the anatomic fracture location. Therefore, the purpose of this study was to (1) evaluate the prevalence of IPV-related UEF; (2) analyze the anatomic location of upper extremity fractures in women due to IPV compared to the distribution of such fractures in similar patients attributing their injury to an accidental fall or accidental striking (control groups); and (3) calculate the odds of specific fracture locations for IPV relative to these control groups.

\section{Methods}

\section{Study design and human subjects}

This retrospective analysis of prospectively collected, publicly available de-identified data was considered exempt by the local Institutional Review Board.

\section{Data source}

Data from the National Electronic Injury Surveillance System All Injury Program (NEISS AIP) were used to identify upper extremity fractures in women. The NEISS is a dataset managed by the US Consumer Product Safety Commission (USCPSC), which collects injury data from $\sim 100$ hospitals (the number varies slightly from year to year) in the USA and its territories having an ED. It was initially designed for injuries due to consumer products. However, not all injuries are from consumer products; thus, the USCPSC selected $\sim 65$ (the number varies slightly from year to year) of these hospitals to obtain data for all injuries, regardless of the association with consumer products. This was designated as the All Injury Program (AIP). This data is in the public domain.

The database includes the date of ED visit, gender/race/ age of the injured patient, diagnosis, disposition from the $\mathrm{ED}$, incident locale, body part injured, perpetrator and type of assault, and hospital size (strata). The body part is classified into five major groups (head/neck, upper trunk, lower trunk, upper extremity, lower extremity), as well as 26 detailed anatomic locations. The hospital strata consist of five categories: four based on size (the total number of ED visits reported by the hospital, which are small [0-16,830], medium [16,831-21,850], large [28,151-41,130], and very large [ $>41,130]$ ), and one encompassing children's hospitals of all sizes. These strata thus encompass both rural and urban hospitals in the USA. An estimated nation-wide 
number of patients are calculated from this weighted, stratified dataset using appropriate statistical techniques [22]. Due to the stratified and weighted nature of the NEISS design, it encompasses and appropriately represents ED visits for the entire USA for all ages, races, rural/urban locations, and both sexes.

\section{Data collection}

NEISS-AIP data for the years 2005 through 2015 was used for this study. These years were chosen because 2015 was the last year in which data was available at the time the study was begun. Data before 2005 was coded differently for many variables, making it difficult to combine the years before 2005 with those afterwards [23]. Injuries due to IPV (following the NEISS structured coding guidelines) were identified by the NEISS AIP codes INTENT_C $=1$ (sexual assault) or 2 (other assault) and $\mathrm{PERP}=1$ (spouse/partner). Sexual assault was included as it is clearly a form of IPV (the NEISS assault definition is given in Appendix 1). Two control groups were selected, those being patients sustaining an accidental fall or those with an accidental striking/being struck by an object. Both control groups did not include any injuries due to motor vehicle accidents, which is a separate category in the NEISS AIP database. They were identified by the NEISS AIP codes INTENT_C $=0$ (unintentional) and ICAUSE_C (immediate cause of the injury) $=6$ (fall) or 7 (struck by/against). As the vast majority of IPV victims are women and there is a difference in the male and female victimization [23], we excluded men from this study. We included only those women 15 through 54 years old as there is a general decline in rates of physical forms of IPV in older women [24]. Patients in study and control groups were split into four subgroups by age decades $(15-24,25-34,35-44$, and $45-54$ years old).

\section{Outcome measures}

The outcome measures were (1) prevalence of IPV-related UEF among all UEF in women and among all IPV-related fractures; (2) distribution of UE fracture anatomic locations in the study and two control groups by each decade of patient age; and (3) odds of a fracture of the finger, forearm, elbow, or shoulder in women with IPV compared to the control groups.

\section{Statistical analysis}

Statistical analyses were performed with SUDAAN 11.0.01 ${ }^{\mathrm{TM}}$ software (RTI International, Research Triangle Park, North Carolina, 2013), accounting for the weighted, stratified nature of the data. Estimated numbers of injuries/ED visits and $95 \%$ confidence intervals [CIs] of the estimates were calculated. Analyses between groups of continuous data were performed with the $t$ test (2 groups) or ANOVA ( 3 or more groups). Differences between groups of categorical data were analyzed by the $\chi^{2}$ test. Demographic predictors of a fracture were determined with multivariate logistic regression analysis, giving an odds ratio (OR) with corresponding $95 \%$ CIs. A $p$ value $<0.05$ was considered statistically significant.

\section{Results}

For both men and women ages 15 through 54 over the 11-year study period, the estimated total number of ED visits for IPV was 1,966,355, for accidental falls 33,189,333 and for accidental striking 31,690,294. The average age for these same three groups was 31.6 years, 34.6 years, and 31.0 years $(p<0.0001)$. The sex distribution was $16.4 \%$ male and $83.6 \%$ female for the IPV group, $47.2 \%$ male and 52.8\% female for the accidental fall group, and $54.7 \%$ female and $45.3 \%$ male for the accidental striking group $(p<0.0001)$. When combing the accidental fall and striking cohorts into one group, the $p$ values remained the same when comparing the IPV to the combined fall and striking control group.

\section{Prevalence of IPV-related upper extremity fractures}

The total number of estimated ED visits for IPV related UEF in women aged 15-54 years accounted for $1.7 \%$ $(40,6722 / 461,368)$ of all UEF and $27.2 \%(40,672 / 149,199)$ of all IPV-related fractures. Fractures of the head and neck were the most common IPV-related fracture, accounting for $52.4 \%(78,283 / 149,199)$ of all IPV fractures (Table 1$)$.

\section{Study cohorts}

Of the 2,461,368 ED visits for UEF in women $15-54$ years old, $50.4 \%(1,241,646)$ were due to a fall, $24.2 \%(595,812)$ were due to accidental striking, and $1.65 \%(40,672)$ were due to IPV. These three cohorts (IPV, and the two control groups - accidental fall and accidental striking) accounted

Table 1 IPV-related fractures in women 15-54 years old

\begin{tabular}{llc}
\hline Region & $\begin{array}{l}\text { Estimated number of ED visits } \\
\text { related to IPV fractures }\end{array}$ & Percentage \\
\hline Head and neck & 78,283 & 52.4 \\
Upper extremity & 40,672 & 27.2 \\
Upper trunk & 14,370 & 9.6 \\
Lower extremity & 13,182 & 8.8 \\
Lower trunk & 2,692 & 1.8 \\
\hline
\end{tabular}


Table 2 Distribution of upper extremity fractures based on reported etiology and location

\begin{tabular}{|c|c|c|c|c|c|c|c|c|c|c|}
\hline \multirow[t]{2}{*}{ Location } & \multicolumn{2}{|l|}{ IPV All } & \multicolumn{2}{|c|}{ IPV Fall } & \multicolumn{2}{|c|}{ IPV Strike } & \multicolumn{2}{|c|}{ Fall Control } & \multicolumn{2}{|c|}{ Strike Control } \\
\hline & $N$ & $\%$ & $N$ & $\%$ & $N$ & $\%$ & $N$ & $\%$ & $N$ & $\%$ \\
\hline Shoulder & 3,740 & 9.2 & 939 & 19.0 & 2,732 & 8.1 & 91,640 & 7.4 & 17,135 & 2.9 \\
\hline Humerus & 1,815 & 4.5 & 411 & 8.3 & 1,270 & 3.7 & 92,762 & 7.5 & 10,776 & 1.8 \\
\hline Elbow & 1,795 & 4.4 & 501 & 10.1 & 1,224 & 3.6 & 156,600 & 12.6 & 11,255 & 1.9 \\
\hline Forearm & 5,082 & 12.5 & 862 & 17.5 & 3,904 & 11.5 & 271,233 & 21.8 & 32,017 & 5.4 \\
\hline Wrist & 5,491 & 13.5 & 952 & 19.3 & 4,293 & 12.7 & 396,074 & 31.9 & 43,700 & 7.3 \\
\hline Hand & 8,781 & 21.6 & 392 & 7.9 & 8,141 & 24.0 & 107,322 & 8.6 & 163,137 & 27.4 \\
\hline Fingers & 13,968 & 34.3 & 879 & 17.8 & 12,314 & 36.3 & 126,016 & 10.1 & 317,796 & 53.3 \\
\hline Total & 40,672 & & 4,936 & & 33,878 & & $1,241,647$ & & 595,816 & \\
\hline
\end{tabular}

for $76.3 \%(1,878,130)$ of all UEF in women $15-54$ years old (Table 2).

\section{Fracture locations in the IPV cohort}

The finger was the most common site, accounting for $34.3 \%$ $(13,968 / 40,672)$ of the IPV UEF. The second most common location was the hand $(21.6 \%)$, followed by the wrist $(13.5 \%)$, forearm $(12.5 \%)$, shoulder $(9.2 \%)$, humerus $(4.5 \%)$, and elbow (4.4\%). Of the 40,672 upper extremity fractures due to IPV, 33,878 (74\%) of the fractures were due to being hit/struck, and 4,936 (12.0\%) of the fractures were due to a fall (Table 3).

\section{Differences between the IPV cohort and control groups}

The finger was the most common fracture location for both IPV (34.3\%) and accidental striking (53.3\%), followed by the hand (21.6\% vs $27.4 \%$ ). However, fractures involving other sites were slightly more common in the IPV cohort than patients with accidental striking. This was noteworthy for the forearm $(12.5 \%$ vs $5.4 \%)$ and shoulder $(9.2 \%$ vs $2.9 \%$ ). In comparison, the wrist $(31.9 \%)$ was the most common site of fracture in patients with a reported history of fall, followed by the forearm $(21.8 \%)$ and elbow $(12.6 \%)$, with finger and hand fractures representing only $10.1 \%$ and $8.6 \%$ of all fall-related upper extremity fractures, respectively (Table 1, Fig. 1). All location differences based on the reported mechanism of injury were significant at $p<0.0001$.

We then compared the location of fractures due to being struck between the IPV and accidental cohorts (Fig. 2). The finger was the most common site of fracture in both groups. However, finger fractures constituted $36.3 \%$ of IPV UEF compared to $53.3 \%$ of UEF in the accidental striking cohort. Wrist $(12.7 \%)$, forearm $(11.5 \%)$, and shoulder $(8.1 \%)$ fractures were more common in the IPV cohort compared to the accidental striking cohort (wrist, 7.3\%; forearm, 5.4\%; and shoulder, $2.9 \%$ ) (Table 2).
Similarly, the wrist remained the most common site of fracture for both IPV fall and accidental fall groups; however, the wrist accounted for $19.3 \%$ of upper extremity fractures related to IPV compared to $31.9 \%$ in accidental falling. The shoulder and wrist were equally frequent (19\%) in the IPV-related fall cohort, while it was the least common site of all fractures in the accidental fall cohort (7.4\%) (Fig. 3).

\section{Differences by age groups and three most common fracture locations}

Among the fractures involving the wrist, hand, and finger, the relative distribution of fractures remained consistent in IPV for the three age decades between 15 and 44 years with finger fractures accounting for 50.2-51\% of such fractures, compared to the dominance of wrist fractures in the fall cohort (53.4-63.0\%) and finger fractures in the accidental striking cohort (55.2-66.0\%). Wrist fractures increased from 35-44-year-old to 45-54-year-old age groups for all three cohorts - from $21.5 \%$ to $28.0 \%$ in IPV, $63 \%$ to $70.9 \%$ in fall, and $7.8 \%$ to $10.4 \%$ in accidental striking (Fig. 3 ).

\section{Odds for etiology based on specific fracture locations}

The odds for specific fracture locations using multivariate logistic regression analysis were determined entering the four age decades and the three cohorts (IPV, falling, striking) as variables into the model (Table 2). The odds of sustaining a finger fracture were greatest in accidental striking $(\mathrm{OR}=9.74$; CI [8.81-10.76]), followed by IPV $(\mathrm{OR}=4.32$; CI [3.41-5.47]) compared to falling (reference). The odds of sustaining a shoulder fracture were greatest in IPV $(\mathrm{OR}=3.65$; CI [2.48-5.39]), followed by a fall $(\mathrm{OR}=2.61$; CI [2.19-3.11]) compared to being accidentally struck (reference). The odds of sustaining a forearm fracture were greatest in a fall $(\mathrm{OR}=4.74$; CI [4.13-5.43], followed by IPV (OR = 2.49; CI [1.87-3.31]) compared to being struck (reference). The odds of sustaining an elbow/humerus fracture were greatest in a fall $(\mathrm{OR}=5.94$; CI [4.92-7.18]) 


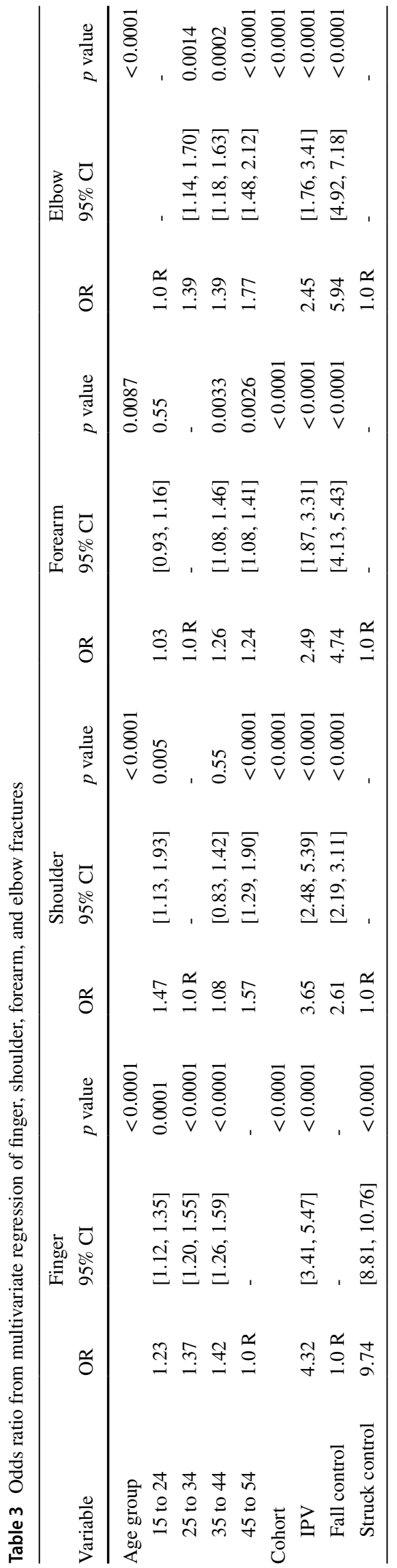

followed by IPV ( $\mathrm{OR}=2.45$; CI [1.76-3.41]) compared to being struck (reference). These were all statistically significant at $p<0.0001$. Although there were effects by age decades (Table 3), they were much less than those by the three cohorts (IPV, accidental striking, accidental fall), with none of the age groups having an OR of $>1.4$ compared to their reference groups. There were no significant effects by hospital size for any of the four fracture locations.

\section{Discussion}

Our results showed that among women 15-54 years old, only $1.7 \%$ of all UEF were reported to result from IPV even though $27.2 \%$ of all IPV related fractures occur in the upper extremity. With one in five women experiencing a severe form of physical violence from an intimate partner in their lifetime, these numbers are consistent with previous reports of significant under-diagnosis [25-30]. The reported cases of IPV represent an insignificant number when compared with the prevalence data, known as the "iceberg" of domestic violence [31]. Barriers to patient disclosure because of shame and fear of retaliation are well documented. Physician factors include lack of awareness, fear of offending the patient and partner, scarcity of time during each encounter, inadequate training to identify IPV, and reluctance to broach an uncomfortable topic [8]. Therefore, it is essential for radiologists to recognize and learn the characteristic injury patterns seen in IPV victims and apply this knowledge to identify those unfortunate victims who are unable to disclose due to multiple barriers in an objective unbiased manner. As our study encompasses the entire USA, all races and several years of data, these results apply to all women with upper extremity fractures, regardless of geographic location or practice type.

Our study found that an accidental fall was the most common injury mechanism for UEF, but the finger accounted for only $10 \%$ of all fall-related UEF. The finger was the most common fracture site for all ages and represented one-third of the IPV and one-half of the accidental striking UEF. We believe this is critical information as victims often misreport accidental falls for their injuries to conceal IPV. Even among IPV patients, more than two-thirds of UEF were sustained due to intentional striking, while a fall occurred in only $12 \%$ of patients. Therefore, a woman presenting with a finger fracture and reporting a dubious history of a fall should be approached for discussion regarding potential IPV as the odds of a finger fracture are 4.32 times greater in IPV than a fall.

We observed a higher prevalence of wrist, forearm, elbow, arm, and shoulder fractures with intentional striking than accidental striking. Because hands and fingers are used for gripping and grasping objects, they are more prone to 
Fig. 1 Distribution of fractures based on the anatomic location for each type of reported injury
Fig. 2 Comparison of location of fractures due to IPV striking versus accidental striking and IPV related fall versus accidental falling
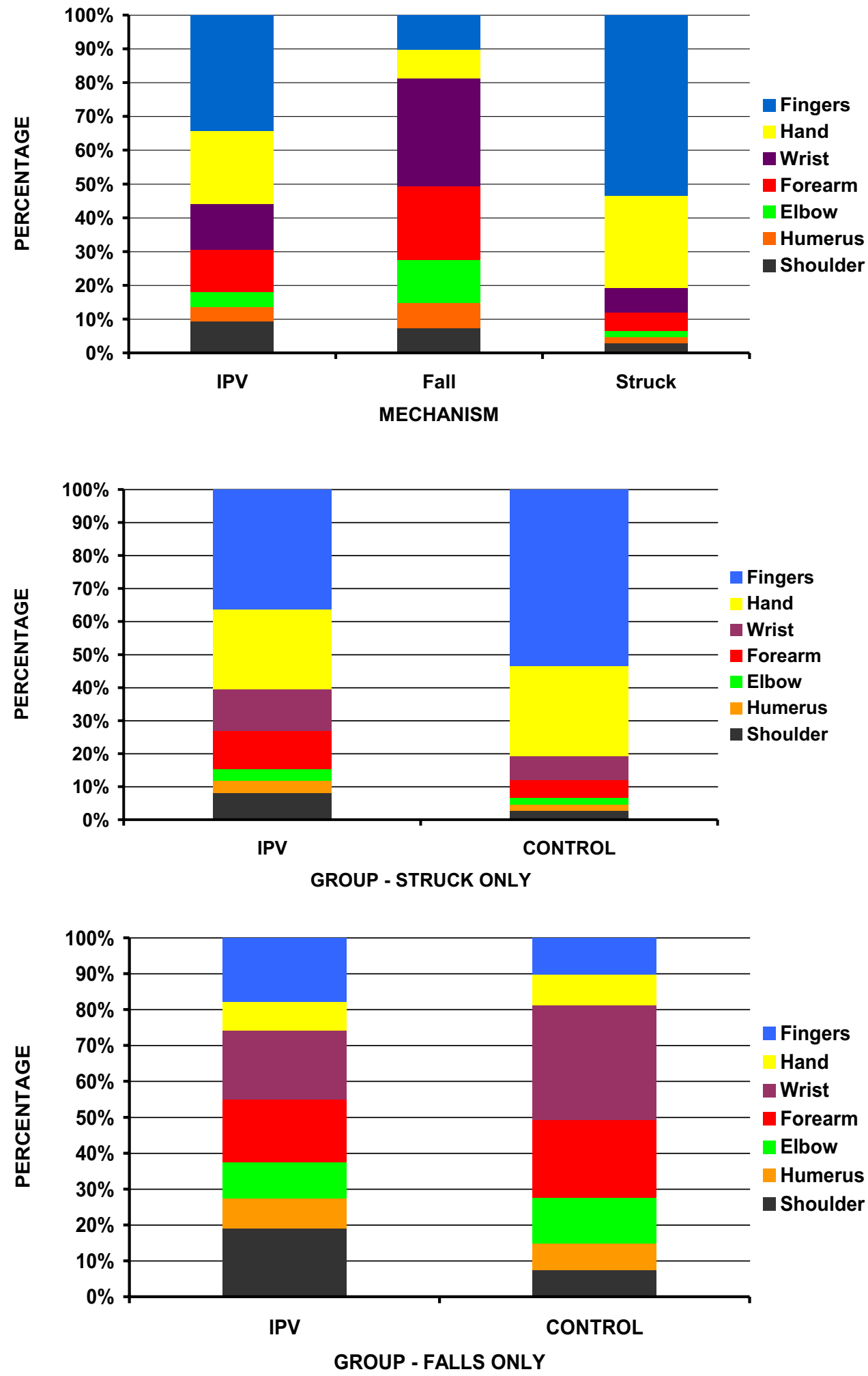

sustain injuries due to accidental striking than the proximal locations of the upper extremity. The forearm, wrist, and hand are more defensive locations being raised to protect the central body. Nightstick fractures, isolated fractures of the ulna, typically located in the mid-diaphysis and usually from a direct blow [20, 32]. Therefore, a woman presenting with a forearm fracture with a vague history of accidental striking should also be approached for a discussion regarding potential IPV as the odds of sustaining a forearm fracture are 2.49 times greater in IPV than in accidental striking. 

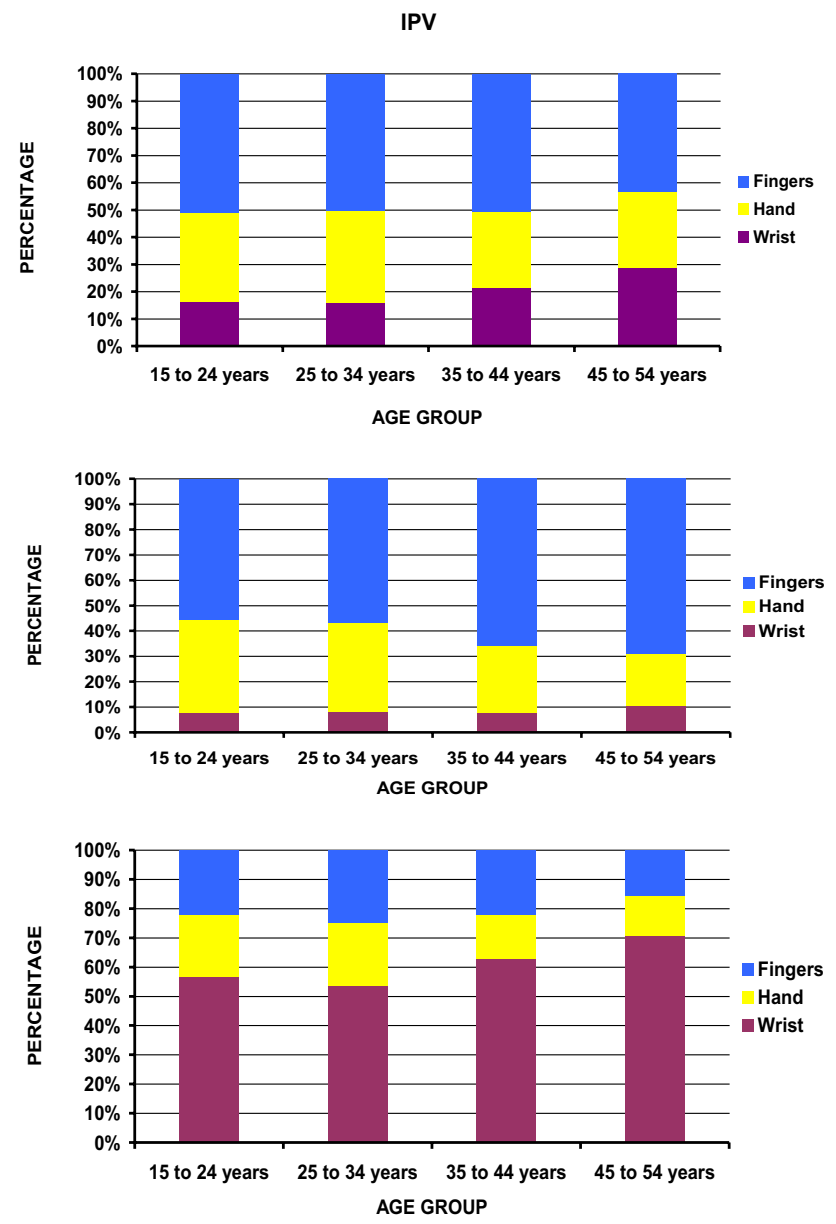

Fig. 3 Location of fractures for each age group in patients reporting IPV, fall control, and accidental striking

Our study showed a higher proportion of shoulder fractures in the IPV cohort than both control groups. While IPVrelated fractures due to falls represented only $12 \%$ of IPV UEF, there was a significantly higher prevalence of shoulder fractures in the IPV fall cohort than the accidental fall cohort (19\% vs. $7.4 \%$ ). In a retrospective review of musculoskeletal manifestations of physical abuse in 263 patients with IPV, the shoulder was the second most common site of fracture/ dislocation after fingers (11/17), with three cases of shoulder dislocation, one humeral, and one clavicle fracture [16]. A higher prevalence of shoulder fractures could be related to direct blow, pushing and shoving victims against walls, or pushing victims to the floor, while the victim is defending and resisting the attacker with her hand and, therefore, unable to take support from an outstretched hand (unlike accidental falls). Therefore, a woman presenting with a shoulder fracture and inconsistent history should be approached for discussion regarding potential IPV as the odds of sustaining a shoulder fracture are 3.65 times more than in accidental striking and 2.61 times more likely than in a fall.
Our study has several limitations, the first and foremost being inadequate information on specific bone involvement. Knowledge of which particular metacarpal or phalangeal bone is fractured might further differentiate IPV from accidental cases; however, we are limited by the information entered into the database. Only a single injury (the most severe one) is recorded for each patient entry in the NEISS database, even if there are multiple injuries. Therefore, we have no information on concomitant or other associated injuries. Similarly, there is no follow-up or access to a previously reported injury. Recurrent and synchronous injury patterns are expected in IPV and might help identify victims [33]. Information on socioeconomic status and other risk factors such as substance use, psychiatric history, and disability is also not included in this database. Finally, we acknowledge that many women in the control groups might be IPV victims but were not forthcoming and the actual odds ratios are likely higher than the provided odds ratios.

In conclusion, the finger is the most common location for IPV-related UEF in women 15-54 years old; however, it is also the most common site of fracture due to accidental striking. The odds of a finger fracture are 4.32 times greater in IPV than falling, of a shoulder fracture 3.65 times greater in IPV than accidental striking and 2.61 times greater in IPV than falling, and of a forearm fracture were 2.45 times greater than accidental striking. Therefore, the possibility of IPV should be discussed with the clinical provider for any woman presenting with a finger fracture and providing a nebulous fall history. Similarly, a woman presenting with a shoulder or forearm fracture and a vague history of accidental striking should also prompt the discussion of IPV. By recognizing characteristic injury locations and unusual injury mechanisms into account, radiologists can facilitate early diagnosis of IPV and prevent more severe and lifethreatening injuries by breaking the cycle of abuse.

\section{Appendix 1: NEISS definition of an assault}

Injury from an act of violence where physical force by one or more persons is used with the intent of causing harm, injury, or death to another person or an intentional poisoning by another person. This category includes perpetrators as well as intended and unintended victims of violent acts (e.g., innocent bystanders). This category excludes unintentional shooting victims (other than those occurring during an act of violence), unintentional drug overdoses, and children or teenagers "horsing" around.

Author contribution Study conception and design: BK, AR, RL, GD, MH, SS, GB, PT.

Data Collection and Analysis: RL. 
Data Interpretation: BK, AR, RL, GD, PT, MH, SS, GB.

All authors discussed the results, provided critical feedback and helped shape the research, analysis and manuscript.

BK wrote the manuscript with input from all authors.

All authors read the manuscript and contributed to the final version of the manuscript.

Funding Gillian Reny Stepping Strong Innovator award, Brigham Health (Protected research time for Bharti Khurana).

Garceau Professorship in Orthopaedic Surgery, Riley Children's Foundation, Indianapolis, IN USA (Protected research time for Randall

T. Loder).

Non-relevant disclosures

Bharti Khurana, MD

GE Health Care Research Grant.

Book Royalties from Cambridge University Press.

Section Editor Royalties, Emergency Radiology, UptoDate, Wolters Kluwer.

Paul Tornetta III, MD.

Receives royalty IP payments from and has pending and issued intellectual property with Smith \& Nephew, owns stock or stock options in $4 \mathrm{Web}$.

Steven E. Seltzer, MD.

GERAF Career Development Fellowship-Travel reimbursement for one board meeting each year.

Academy of Radiology Research-Volunteer position as Senior Fellow for Health and Science Policy.

George Dyer, MD.

Grants from Depuy Synthes and Stryker.

\section{Declarations}

Conflict of interest The authors declare that they have no conflict of interest.

\section{References}

1. Sharon G Smith, Xinjian Zhang, Kathleen C Basile, Melissa T Merrick, Jing Wang, Marcie-jo Kresnow, et al. 2015 NISVS data brieflviolence preventionlinjury center|CDC 2019. https://www. cdc.gov/violenceprevention/datasources/nisvs/2015NISVSdatabr ief.html (accessed 10 May 2020)

2. Garcia-Moreno C, Jansen HA, Ellsberg M, Heise L, Watts CH (2006) Prevalence of intimate partner violence: findings from the WHO multi-country study on women's health and domestic violence. Lancet 368:1260-1269. https://doi.org/10.1016/S01406736(06)69523-8

3. Goldberg WG, Tomlanovich MC (1984) Domestic violence victims in the emergency department. New findings. JAMA 251:3259-3264

4. Dicola D (2016) Intimate partner violence. Intim Partn Violence 94:6

5. Wadman MC, Muelleman RL (1999) Domestic violence homicides: ED use before victimization. Am J Emerg Med 17:689-691. https://doi.org/10.1016/S0735-6757(99)90161-4

6. McLeer SV, Anwar R (1989) A study of battered women presenting in an emergency department. Am J Public Health 79:65-66

7. George E, Phillips CH, Shah N, Lewis-O'Connor A, Rosner B, Stoklosa HM et al (2019) Radiologic findings in intimate partner violence. Radiology 291:62-69. https://doi.org/10.1148/radiol. 2019180801
8. Elliott L, Nerney M, Jones T, Friedmann PD (2002) Barriers to screening for domestic violence. J Gen Intern Med 17:112-116. https://doi.org/10.1046/j.1525-1497.2002.10233.x

9. Matteoli M, Piacentino D, Kotzalidis GD, Serata D, Rapinesi C, Angeletti G et al (2016) The clinical and radiological examination of acute intimate partner violence injuries: a retrospective analysis of an Italian cohort of women. Violence Vict 31:85102. https://doi.org/10.1891/0886-6708.VV-D-14-00107

10. Reis BY, Kohane IS, Mandl KD (2009) Longitudinal histories as predictors of future diagnoses of domestic abuse: modelling study. BMJ 339:b3677-b3677. https://doi.org/10.1136/bmj. b3677

11. Joseph B, Khalil M, Zangbar B, Kulvatunyou N, Orouji T, Pandit $\mathrm{V}$ et al (2015) Prevalence of domestic violence among trauma patients. JAMA Surg 150:1177-1183. https://doi.org/10.1001/ jamasurg.2015.2386

12. Gosangi B, Park H, Thomas R, Gujrathi R, Bay CP, Raja AS et al (2020) Exacerbation of physical intimate partner violence during COVID-19 pandemic. Radiology 202866. https://doi.org/10.1148/ radiol.2020202866

13. Halpern LR, Perciaccante VJ, Hayes C, Susarla S, Dodson TB (2006) A protocol to diagnose intimate partner violence in the emergency department. J Trauma 60:1101-1105. https://doi.org/ 10.1097/01.ta.0000218247.58465.db

14. Campbell JC (2002) Health consequences of intimate partner violence. Lancet 359:6

15. Alessandrino F, Keraliya A, Lebovic J, Mitchell Dyer GS, Harris MB, Tornetta $P$ et al (2020) Intimate partner violence: a primer for radiologists to make the "invisible" visible. Radiogr Rev Publ Radiol Soc N Am Inc 200010. https://doi.org/10.1148/rg.20202 00010

16. Bhandari M, Dosanjh S, Tornetta P, Matthews D (2006) Musculoskeletal manifestations of physical abuse after intimate partner violence: J Trauma Inj Infect. Crit Care 61:1473-1479. https:// doi.org/10.1097/01.ta.0000196419.36019.5a

17 Loder RT, Momper L (2020) Demographics and fracture patterns of patients presenting to US emergency departments for intimate partner violence. JAAOS Glob Res Rev 4:e20.00009. https://doi. org/10.5435/JAAOSGlobal-D-20-00009

18. PRAISE Investigators, Sprague S, Bhandari M, Della Rocca GJ, Goslings JC, Poolman RW et al (2013) Prevalence of abuse and intimate partner violence surgical evaluation (PRAISE) in orthopaedic fracture clinics: a multinational prevalence study. Lancet Lond Engl 382:866-876. https://doi.org/10.1016/S0140-6736(13) $61205-2$

19. Thomas R, Dyer GSM, Tornetta Iii P, Park H, Gujrathi R, Gosangi B et al (2021) Upper extremity injuries in the victims of intimate partner violence. Eur Radiol. https://doi.org/10.1007/ s00330-020-07672-1

20. Khurana B, Sing D, Gujrathi R, Keraliya A, Bay CP, Chen I et al (2021) Recognizing isolated ulnar fracture as a potential marker for intimate partner violence. J Am Coll Radiol JACR. https://doi. org/10.1016/j.jacr.2021.03.006

21. Shepherd JP, Shapland M, Pearce NX, Scully C (2016) Pattern, severity and aetiology of injuries in victims of assault. J R Soc Med. https://doi.org/10.1177/014107689008300206

22. Schroeder T, Ault K. The NEISS Sample (design and implementation) 1997 to Present n.d.:37. Division of Hazard and Injury Data Systems U.S. Consumer Product Safety Commission June 2001

23. Kolbe V, Büttner A. (2020) Domestic Violence Against Men-Prevalence and Risk Factors. Dtsch Arztebl Int 3:117(31-32):534-541

24. Pathak N, Dhairyawan R, Tariq S (2019) The experience of intimate partner violence among older women: a narrative review. Maturitas 121:63-75. https://doi.org/10.1016/j.maturitas.2018.12. 011 
25. Yau RK, Stayton CD, Davidson LL (2013) Indicators of intimate partner violence: identification in emergency departments. J Emerg Med 45:441-449. https://doi.org/10.1016/j.jemermed. 2013.05.005

26. Rhodes KV, Kothari CL, Dichter M, Cerulli C, Wiley J, Marcus S (2011) Intimate Partner Violence identification and response: time for a change in strategy. J Gen Intern Med 26:894-899. https://doi. org/10.1007/s11606-011-1662-4

27 Abbott J, Johnson R, Koziol-McLain J, Lowenstein SR (1995) Domestic violence against women. Incidence and prevalence in an emergency department population. Jama 273:1763-1767. https:// doi.org/10.1001/jama.273.22.1763

28. Dearwater SR, Coben JH, Campbell JC, Nah G, Glass N, McLoughlin E et al (1998) Prevalence of intimate partner abuse in women treated at community hospital emergency departments. JAMA 280:433-438. https://doi.org/10.1001/jama.280.5.433

29. Lipsky S, Caetano R, Field CA, Bazargan S (2004) Violencerelated injury and intimate partner violence in an urban emergency department. J Trauma 57:352-359. https://doi.org/10.1097/01.ta. 0000142628.66045.e2

30. Walton MA, Murray R, Cunningham RM, Chermack ST, Barry $\mathrm{KL}$, Booth BM et al (2009) Correlates of intimate partner violence among men and women in an inner city emergency department. J Addict Dis 28:366-381. https://doi.org/10.1080/1055088090 3183018

31. Palermo T, Bleck J, Peterman A (2014) Tip of the iceberg: reporting and gender-based violence in developing countries. Am J Epidemiol 179:602-612. https://doi.org/10.1093/aje/kwt295

32 Ali M, Clark DI, Tambe A (2019) Nightstick fractures, outcomes of operative and non-operative treatment. Acta Med (Hradec Kralove) 62:19-23. https://doi.org/10.14712/18059694.2019.41

33. Khurana B, Seltzer SE, Kohane IS, Boland GW (2019) Making the 'invisible' visible: transforming the detection of intimate partner violence. BMJ Qual Saf bmjqs-2019-009905. https://doi.org/ 10.1136/bmjqs-2019-009905

Publisher's note Springer Nature remains neutral with regard to jurisdictional claims in published maps and institutional affiliations. 